\title{
DESEMPENHO DE CORDEIROS LACTENTES RECEBENDO PROBIÓTICOS EM COMEDOUROS PRIVATIVOS
}

\author{
(Performance of lambs receiving probiotics in creep feeding)
}

\author{
PINHEIRO, R. S. B. ${ }^{1}$; SOBRINHO, A. G. S. ${ }^{2}$; YAMAMOTO, S. M. ${ }^{3}$ \\ ${ }^{1}$ Doutorando em Zootecnia da FMVZ/Unesp, Botucatu, SP. \\ 2Departamento de Zootecnia da FCAV/Unesp, Jaboticabal, SP. \\ ${ }^{3}$ Pós-doutoranda do Programa de Pós-graduação em Zootecnia da Universidade Estadual de Maringá, PR.
}

\begin{abstract}
RESUMO - O presente estudo teve como objetivo avaliar o desempenho de 40 cordeiros $7 / 8$ lle de France 1/8 Ideal (20 machos não castrados e 20 fêmeas) criados com acesso a comedouros privativos, até atingirem $17 \mathrm{~kg}$ de peso corporal. Os animais foram distribuídos em diferentes tratamentos, recebendo dietas (D) isoprotéicas e isoenergéticas, com diferentes teores de probióticos (D1= 0\%, D2=0,8\% e D3=1,2\%). Foram analisados peso ao nascer, peso aos 30 dias após o nascimento e ganho de peso diário nas fases materno-dependente (primeira à quarta semana) e na materno-independente (quinta à oitava semana), idade ao desmame e ganho de peso diário. O consumo de matéria seca dos cordeiros nas D1, D2 e D3 foi de 0,27, 0,26 e 0,27 kg/dia, respectivamente. $\mathrm{Na}$ fase materno-dependente, não houve interação e diferenças entre dieta e sexo no peso ao nascer $(3,30 \mathrm{~kg})$, peso aos 30 dias $(11,79 \mathrm{~kg})$ e ganho médio de peso diário $(0,28$ $\mathrm{kg}$ ). Na fase materno-independente, também não houve diferenças na idade ao desmame (53 dias) e ganho de peso diário $(0,26 \mathrm{~kg})$ entre dieta e sexo. Concluiu-se que a inclusão de probióticos na dieta de cordeiros lactentes (machos não castrados e fêmeas) não melhorou o desempenho dos mesmos.
\end{abstract}

Palavras-chave: consumo; ganho de peso; ovinos; promotor de crescimento.

\begin{abstract}
The presente study was carried out to evaluate the performance of 40 lambs $7 / 8$ lle de France $1 / 8$ Ideal (20 not castrated males and 20 females) raised with access to creep feeding, until get $17 \mathrm{~kg}$ of corporeal weight. The animals were divided in treatments, receiving isoproteic and isoenergetic diets, with diferent levels of probiotics (D1 $=0 \%, D 2=0,8 \%$ e $D 3=12 \%)$. They were analysed in regart to born weight, weight at 30 days and weight gain at maternal dependent (from 1 th to 4 th weeks) and maternal independent (from 5th to 8th week), the age of wean and daily weight gain. The average intake of dry matter by the lambs at D1, D2 and D3 was $0,27,0,26$ and $0,27 \mathrm{~kg} /$ day, respectively. On maternal dependent phase, there was no interaction and differences between diet and sex in the born weight $(3.30 \mathrm{~kg})$, weight at 30 days of age $(11.79 \mathrm{~kg})$ and in the average daily weight gain $(0.28 \mathrm{~kg})$. On maternal independent phase, there were no differences in the age of weam (53 days) and daily weight gain $(0,26 \mathrm{~kg}$ ) between diet and sex. It was concluded that probiotics inclusion in the diet of the suckling lambs (males not castrated and females) didn't improve their performance.
\end{abstract}

Key-words: growth promoter; intake; lambs; weight gain. 
Desempenho de cordeiros lactentes recebendo probióticos em comedouros privativos

\section{INTRODUÇÃO}

O uso de técnicas que melhorem o desempenho de cordeiros tem despertado o interesse dos criadores, no intuito de diminuir o déficit de carne ovina frente ao crescimento do mercado. A nutrição é utilizada como estratégia para aumentar os índices de produção e os promotores de crescimento podem contribuir para meIhorar o desempenho de ruminantes em crescimento (ALVES et al., 2004).

O uso de antibióticos e hormônios em dietas é uma prática que vem sendo banida, pois sua utilização pode deixar resíduos nos produtos de origem animal. Os probióticos vem sendo utilizados como produtos alternativos, atuando na manutenção do equilíbrio da flora intestinal, em harmonia com a função digestiva e a saúde do animal. Segundo VANBELLE et al. (1990), probióticos são microrganismos vivos, que após consumidos, são capazes de estabelecerem-se no trato gastrintestinal mantendo ou aumentando a microbiota benéfica, além de prevenir a colonização de microrganismos patogênicos, assegurando melhor utilização dos nutrientes.

Em sistemas tradicionais de manejo, o desenvolvimento dos cordeiros é comprometido pelas inadequadas condições de desmame às quais os mesmos são submetidos, observando-se diminuição na produção de leite das ovelhas, entre a $3^{\mathrm{a}}$ e a $4^{\mathrm{a}}$ semana após parto (MARQUES et al., 2003). De acordo com RAMSEY et al. (1994), este é o momento em que os cordeiros aumentam suas exigências nutricionais, necessitando de outras fontes de nutrientes.

O uso de comedouros privativos permite adaptação mais rápida dos cordeiros ao alimento sólido, diminuindo a dependência pelo leite materno e aumentando o peso ao desmame (VILLAS BÔAS, 2000), resultando em maior eficiência do sistema de produção.
O experimento objetivou avaliar o desempenho de cordeiros lactentes de diferentes sexos (machos não castrados e fêmeas), nas fases materno dependente e independente, recebendo diferentes teores de probióticos até o desmame dos mesmos.

\section{MATERIAL E MÉTODOS}

O experimento foi conduzido no Setor de Ovinocultura, pertencente ao Departamento de Zootecnia da FCAV/Unesp, Campus de Jaboticabal, SP, nos meses de março a junho de 2004.

Utilizou-se 40 cordeiros $7 / 8$ lle de France 1/8 Ideal (20 machos não castrados e 20 fêmeas) recémnascidos e manejados até o primeiro dia em piquete maternidade, quando foram transferidos com suas respectivas mães, para três piquetes com pastagem de Tifton - 85 (Cynodon dactylon) com $2500 \mathrm{~m}^{2}$ cada. $\mathrm{Na}$ área sombreada de descanso das ovelhas, foram disponibilizados em cada piquete um local coberto de 2,30 $\mathrm{m} \times 2,30 \mathrm{~m}$, com mourões distanciados 20 $\mathrm{cm}$ um do outro, contendo em seu interior cochos de madeira com acesso privativo aos cordeiros.

Os animais receberam dietas isoprotéicas e isoenergéticas, com diferentes teores de probióticos, composto por Lactobacillus acidophilus, Streptococcus faecium", "Bacillus cereus, Ruminobacter amylophitum, Ruminobacter succinogenes, Succinovibrio dextrinosolvens e veículo de carbonato de cálcio. Os tratamentos constituíram em $0 \%$ (controle), 0,8\% e $1,2 \%$ de probióticos, cujas composições percentual e bromatológica foram realizadas conforme metodologias descritas por SILVA e QUEIROZ (2002) e que se encontram na TABELA 1.

As dietas foram fornecidas uma vez ao dia, às sete horas da manhã, com ajuste diário do consumo

TABELA 1 - COMPOSIÇÕES PERCENTUAL E BROMATOLÓGICA DAS DIETAS EXPERIMENTAIS DE CORDEIROS LACTENTES EM COMEDOUROS PRIVATIVOS.

\begin{tabular}{|c|c|c|c|}
\hline \multirow[t]{2}{*}{ INGREDIENTE (\%) } & \multicolumn{3}{|c|}{ DIETA $^{a}$} \\
\hline & D1 & D2 & D3 \\
\hline GRÃO DE MILHO MOÍDO & 54,60 & 54,60 & 54,60 \\
\hline FARELO DE TRIGO & 18,00 & 18,00 & 18,00 \\
\hline FARELO DE SOJA & 24,50 & 24,50 & 24,50 \\
\hline CALCÁRIO CALCITICO & 1,80 & 1,80 & 1,80 \\
\hline FOSFATO BICÁLCICO & 0,10 & 0,10 & 0,10 \\
\hline SAL IODADO & 0,50 & 0,50 & 0,50 \\
\hline SUPLEMENTO MINERAL ${ }^{b}$ & 0,50 & 0,50 & 0,50 \\
\hline \multicolumn{4}{|l|}{ NUTRIENTES (\%) } \\
\hline MATÉRIA SECA & 90,23 & 89,63 & 89,85 \\
\hline MATÉRIA MINERAL & 7,80 & 8,90 & 9,38 \\
\hline PROTEÍNA BRUTA & 23,78 & 23,60 & 23,64 \\
\hline EXTRATO ETÉREO & 2,50 & 2,57 & 2,55 \\
\hline
\end{tabular}

${ }^{\mathrm{a}} \mathrm{D} 1=0 \%$ (controle), D2 $=0,8 \%$ de probióticos na dieta e D3 $=1,2 \%$ de probióticos na dieta. ${ }^{\text {bSuplemento }}$ mineral: cálcio $22,88 \%$; fósforo $5,8 \%$; enxofre $2,4 \%$; sódio $7,2 \%$; cloro $11,6 \%$; ferro 1.600 ppm; flúor 480 ppm; cobre 250 ppm; zinco 6.000 ppm; selênio 15 ppm; iodo 200 ppm; cobalto 100 ppm e manganês 900 ppm. 
para permitir $20 \%$ de sobras. Os cordeiros foram pesados ao nascer e aos 30 dias de idade e permaneceram em regime de pasto com as ovelhas durante o período experimental, até atingirem $17 \mathrm{~kg}$, peso corporal pré-estabelecido para o desmame.

Os dados de desempenho foram agrupados em duas fases: fase materno dependente, que corresponde do nascimento à quarta semana de idade e a fase materno-independente, da quinta à oitava semana de idade, de acordo com SILVA SOBRINHO (1992).

O delineamento experimental foi o inteiramente casualizado em esquema fatorial $3 \times 2$ (três dietas e dois sexos). A análise de variância foi conduzida segundo procedimentos do programa estatístico Statistical Analysis System (SAS, 1996), versão 6.12, considerando-se o nível de significância de 5\%. O modelo estatístico utilizado foi:

Yijh $=m+\mathrm{Ti}+\mathrm{Cj}+(\mathrm{TC}) \mathrm{ij}+\mathrm{Eijh}$, sendo:

Yijh = valor observado para a característica analisada; $\mathrm{m}=$ média geral; $\mathrm{Ti}=$ efeito da dieta experimental i, com i variando de 1 a 3 ; $\mathrm{Cj}=$ efeito do sexo do animal $\mathrm{j}$, com $\mathrm{j}$ variando de 1 a 2 ; (TC)ij = efeito da interação (dieta experimental x sexo do animal); Eijh = erro experimental.

\section{RESULTADOS E DISCUSSÃO}

$\mathrm{Na}$ TABELA 2 consta o consumo de matéria seca dos cordeiros lactentes e os parâmetros de desempenho, nas fases materno-dependente.

TABELA 2 - CONSUMO DAS DIETAS EXPERIMENTAIS E DESEMPENHO DE CORDEIROS NAS FASES MATERNO-DEPENDENTE (DO NASCIMENTO À QUARTA SEMANA DE IDADE).

\begin{tabular}{lcccccccc}
\hline VARIÁVEL (kg) & \multicolumn{3}{c}{ DIETA $^{a}$} & & \multicolumn{3}{c}{ SEXO } & CVb $(\%)$ \\
\cline { 2 - 3 } & D1 & D2 & D3 & & MACHO & FÊMEA & \\
\hline CONSUMO DIÁRIO & 0,27 & 0,26 & 0,27 & & & & \\
PESO AOS 30 DIAS & 3,25 & 3,37 & 3,28 & & 3,28 & 3,31 & 17,75 \\
GANHO DE PESO & 12,06 & 11,65 & 11,67 & & 11,97 & 11,66 & 17,92 \\
GANHO DE PESO DIÁRIO & 0,29 & 0,27 & 0,28 & & 0,29 & 0,28 & 20,68 \\
\hline
\end{tabular}

${ }^{\mathrm{a}} \mathrm{D} 1=0 \%$ (controle), $\mathrm{D} 2=0,8 \%$ de probióticos na dieta e $\mathrm{D} 3=1,2 \%$ de probióticos na dieta. Médias não diferem pelo teste Tukey $(P>0,05)$. ${ }^{\mathrm{b}} \mathrm{CV}=$ coeficiente de variação.

O consumo de matéria seca pelos cordeiros do nascimento ao desmame, foi $0,27,0,26$ e $0,27 \mathrm{~kg} / \mathrm{dia}$, nas dietas contendo $0 \%$ (controle), $0,8 \%$ e $1,2 \%$ de probióticos, respectivamente. Estes valores estão de acordo com o de $0,23 \mathrm{~kg} / \mathrm{dia}$ citado por MARQUES et al. (2003), ao avaliarem o desempenho de cordeiros com a mesma composição genética, com acesso a concentrado com $20 \%$ de proteína bruta em comedouros seletivos. GONÇALVES et al. (2000), ao avaliarem o desempenho de bezerros do nascimento aos 60 dias de idade, recebendo volumoso e concentrado à vontade nos tratamentos leite integral (controle), leite integral + probiótico em pó $(0,05 \mathrm{~kg} / \mathrm{animal} / \mathrm{dia})$ e leite integral + probiótico em pó $(0,05 \mathrm{~kg} / \mathrm{animal} / \mathrm{dia})+$ pasta probiótica (10 mL/animal/dia), também não encontraram diferenças $(P>0,05)$ no consumo de matéria seca entre os tratamentos, cuja média foi $0,49 \mathrm{~kg} /$ animal/dia.

$\mathrm{Na}$ fase materno dependente, não houve interação e diferenças $(P>0,05)$ entre dieta e sexo quanto ao peso ao nascer $(3,30 \mathrm{~kg})$, peso aos 30 dias $(11,79 \mathrm{~kg})$ e ganho médio de peso diário $(0,28 \mathrm{~kg})$, conforme TABELA 2. MOTTA et al. (2000) ao estudarem o efeito do sexo em cordeiros utilizando comedouros privativos, não constataram diferenças nos ganhos de peso diário para machos e para fêmeas, estando de acordo com os resultados deste trabalho, que também não verificou diferenças entre machos não castrados e fêmeas, para o ganho de peso diário tanto na fase materno-dependente como na independente (TABELA 2 e 3).

As médias dos valores da idade ao desmame dos cordeiros e o ganho de peso diário na fase maternoindependente (da quinta à oitava semana de idade) encontram-se na TABELA 3.

$\mathrm{Na}$ fase materno independente, não houve diferenças $(P>0,05)$ quanto à idade ao desmame entre os tratamentos (52,98 dias) e também para o ganho de peso diário $(0,26 \mathrm{~kg})$, conforme TABELA 3. SILVA SOBRINHO et al. (2004) ao avaliarem cordeiros nesta mesma fase (da quinta à oitava semana de idade), criados com acesso a comedouros privativos, recebendo sal proteinado com 18 e $22 \%$ de proteína bruta, encontraram valores semelhantes de ganho de peso diário $(0,25 \mathrm{~kg})$ e idade ao desmame (55 dias). 
Desempenho de cordeiros lactentes recebendo probióticos em comedouros privativos

TABELA 3 - DESEMPENHO DOS CORDEIROS NA FASE MATERNO INDEPENDENTE (DA QUINTA À OITAVA SEMANA DE IDADE).

\begin{tabular}{|c|c|c|c|c|c|c|}
\hline \multirow[t]{2}{*}{ VARIÁVEL } & \multicolumn{3}{|c|}{ DIETA $^{a}$} & \multicolumn{2}{|c|}{ SEXO } & \multirow[t]{2}{*}{$c V^{b}$} \\
\hline & D1 & $\mathrm{D} 2$ & D3 & $\mathrm{MACHO}$ & FÊMEA & \\
\hline IDADE AO DESMAMEC $^{\mathrm{C}}$ & 53,25 & 52,55 & 53,16 & 52,03 & 53,66 & 20,52 \\
\hline GANHO DE PESO DIÁRIOd & 0,22 & 0,30 & 0,25 & 0,26 & 0,25 & 41,41 \\
\hline
\end{tabular}

${ }^{\mathrm{a} D} 1=0 \%$ (controle), D2 $=0,8 \%$ de probióticos na dieta e D3 $=1,2 \%$ de probióticos na dieta. Médias não diferem pelo teste Tukey $(P>0,05)$. bCV = coeficiente de variação em porcentagem. cldade ao desmame $(\mathrm{kg})$. dGanho de peso diário $(\mathrm{kg})$.

CAMPOS et al. (2004) citaram que o desempenho de ovinos recebendo dieta sem aditivos, foi $20,75 \%$ inferior ao dos que receberam dietas com culturas de levedura e bactéria, discordando dos resultados deste estudo (TABELA 2 e 3 ).

\section{CONCLUSÕES}

O uso de probióticos não afetou o desempenho de cordeiros lactentes nas fases materno dependente e independente, com acesso a comedouros privativos.

Os parâmetros estudados foram semelhantes entre os diferentes sexos (machos não castrados e fêmeas) estudados.

\section{REFERÊNCIAS}

ALVES, J.B.; ISEPON, O.J.; BERGAMASHINE, A.F. Efeitos de aditivos alimentares enzimáticos contendo probiótico no desempenho de bovinos Guserá em confinamento. In: REUNIÃO ANUAL DA SOCIEDADE BRASILEIRA DE ZOOTECNIA, 41. 2004, Campo Grande, Anais... Campo Grande: Sociedade Brasileira de Zootecnia, 2004, 1 CD-ROM.

CAMPOS, C.F.A.; ORSINE, G.F.; OLIVEIRA, E.R.; FALEIRO, J.S. Uso de probiótico para bezerras leiteiras mestiças. In: CONGRESSO NACIONAL DE ZOOTECNIA, 9. 2004, Brasília, Anais... Brasília: Zootec, 2004, 1 CD-ROM.

GONÇALVES, G.D.; SANTOS, G.T.; RIGOLON, L.P.; DAMASCENO, J.C.; RIBAS, N.P.; VEIGA, D.R.; MARTINS, E.N. Influência da adição de probióticos na dieta sobre o estado sanitário e desempenho de bezerros da raça Holandesa. Brazilian Journal of Veterinary Research and Animal Science, v.37, n.1, p. 74-78, 2000.
MARQUES, C.A.T.; SILVASOBRINHO,A.G.; GONZAGA NETO, S.; ROMBOLA, L.G.; LEÃO, A.G. Desempenho de cordeiros criados com acesso a comedouros seletivos (creep feeding) e produção de leite das ovelhas. In: REUNIÃO ANUAL DA SOCIEDADE BRASILEIRA DE ZOOTECNIA, 40. 2003, Santa Maria, Anais... Santa Maria: Sociedade Brasileira de Zootecnia, 2003, 1 CDROM.

MOTTA, O.S.; PIRES, C.C.; SILVA, J.H.S.; ROSA, G.T. Efeito do sexo, peso de abate e uso de "creep feeding" sobre o ganho de peso de ovinos do nascimento ao abate. In: REUNIÃO ANUAL DA SOCIEDADE BRASILEIRA DE ZOOTECNIA, 37. 2000, Viçosa, Anais... Viçosa: Sociedade Brasileira de Zootecnia, 2000, p. 39-46.

RAMSEY, W.S.; HATFIELD,P.G.; WALLACE, J.D.; SOTHWARD,G.M. Relationships among ewe milk production and ewe and lamb forage intake in Targhee ewes nursing single or twin lambs. Journal of Animal Science, v.72, n. 4, p.811-816, 1994.

SAS INSTITUTE. User's Guide to Statistics. Version 6.12. Cary, USA: North Caroline State University, 1996.

SILVA SOBRINHO, A.G. Producción y composición quimica de la leche mediante metodos doble pesada y oxitocina en ovejas Manchegas com tipos de parto simple y doble. Córdoba, 1992. 65p. Tese (Postdoctoral) Universidad de Córdoba.

SILVA SOBRINHO, A.G.; VANTINI FILHO, L.R.; YAMAMOTO, S.M.; PINHEIRO, S.B.P.;ANDRADE, A.T. Desempenho de cordeiros recebendo sal proteinado com diferentes teores protéicos em comedouros privativos (creep feeding). In: REUNIÃO ANUAL DA SOCIEDADE BRASILEIRADE ZOOTECNIA, 41.2004, Campo Grande. Anais... Campo Grande: Sociedade 
Brasileira de Zootecnia, 2004, 1 CD-ROM.

SILVA, D.J.; QUEIROZ, A.C. Análise de Alimentos: métodos químicos e biológicos. 5. (Ed). Viçosa: Imprensa Universitária, 2002. 235p.

VANBELLE, M.; TELLER, E.; FOCANT, M.. Probiotics in animal nutrition: a review. Archives of Animal Nutrition, v.40, n.7, p.543-567, 1990.

VILLAS BÔAS, A.S. Manejo pré-desmame de cordeiros superprecoces e desempenho de ovelhas. Botucatu, 2000. 70p. Tese (Doutorado em Zootecnia) - Faculdade de Medicina Veterinária e Zootecnia, Universidade Estadual Paulista.

Recebido para publicação:

$30 / 05 / 2007$

Aprovado:

$09 / 11 / 2007$ 